\title{
Rupture of entangled polymeric liquids in elongational flow with dissipation*
}

\author{
Yogesh M. Joshi and Morton M. Denn ${ }^{\text {a) }}$ \\ Benjamin Levich Institute for Physico-Chemical Hydrodynamics and Department \\ of Chemical Engineering, City College of New York, CUNY, 1-M Steinman \\ Hall, 140th Street at Convent Avenue, New York, New York 10031
}

(Received 30 July 2003; final revision received 12 January 2004)

\section{Synopsis}

The scaling theory for rupture of entangled polymeric liquids, which is based on a critical recoverable strain [Joshi, Y. M., and M. M. Denn, J. Rheol. 47, 291-298 (2003)], is extended to include the viscoelastic regime, where the total strain at rupture is a decreasing function of the elongation rate. Rupture can be predicted in both the viscoelastic and rubbery regimes using the scaling theory together with a constitutive equation for the stress in the entangled liquid. The scaling theory with the Wagner model captures the essential features of the available experimental data, and the quantitative agreement is reasonable. (c) 2004 The Society of Rheology.

[DOI: $10.1122 / 1.1687788]$

\section{INTRODUCTION}

Rupture in elongational flow of entangled polymeric liquids at high stresses is superficially similar to cohesive failure in solids. Rupture has recently been reviewed by Joshi and Denn (2004), following earlier reviews by Malkin and Petrie (1997) and Ghijsels and co-workers (1997). There are four regimes for filament failure [Vinogradov et al. (1975)]: The first occurs at low elongation rates, where the viscous flow is dominant, the total strain at failure increases with elongation rate, breakup occurs because of necking or a surface tension-driven instability, and strain recovery is negligible. Rupture occurs in the second and third regimes. Viscous flow is still important in the second (viscoelastic) regime, where the total strain at rupture decreases with increasing elongation rate but strain recovery increases. Recoverable elastic strain dominates in the third (rubbery) regime, where the total strain at rupture increases with increasing elongation rate. The final regime, at very high elongation rate, is glass-like, and the total strain at rupture again decreases with elongation rate.

We recently developed a scaling theory for rupture [Joshi and Denn (2003)] in which catastrophic failure occurs when the frictional force on an entangled chain can no longer balance the tension in the chain. Rupture is identified with a critical value of the recoverable strain $\varepsilon_{R}$, given by the solution to

$$
\exp \left(3 \varepsilon_{R}\right)-\exp \left(2 \varepsilon_{R}\right)=\beta \frac{4 \pi^{2}}{5 N_{e}} N^{1 / 2} W e_{\mathrm{Rep}} .
$$

\footnotetext{
a)Author to whom correspondence should be addressed; electronic mail: denn@ccny.cuny.edu

*The editorial process for this manuscript was carried out by Editorial Board member Ronald Larson.
} 
Here, the Weissenberg number $W e_{\text {Rep }}=\dot{\varepsilon} \tau_{d}$ is the product of the reptation time $\tau_{d}$ and the constant elongation rate $\dot{\varepsilon}, N_{e}$ is the average number of monomers between neighboring entanglements, and $N$ is the number of entanglements per molecule. $\beta$ is a scaling parameter of order unity, reflecting the approximations inherent in the estimate of the chain force balance. As developed in Joshi and Denn (2003), the scaling model was limited to the rubbery region, where the total strain at rupture is nearly equal to the recoverable strain, and $\beta$ was taken to be unity. We show here how to extend the theory to the viscoelastic regime, in which dissipation is important.

\section{THEORY}

Incorporating dissipation in order to apply the scaling analysis to Vinogradov's second regime of failure is straightforward in principle. Equation (1) is used to calculate the recoverable portion of the strain at failure for a given elongation rate. A constitutive equation for the entangled melt is then used to compute the total elongational strain corresponding to the recoverable strain computed from Eq. (1); the time to rupture, which is simply the ultimate strain divided by the elongation rate, also follows from this calculation. The methodology is applicable to any constitutive equation of the Rivlin-Sawyer form (including $\mathrm{K}-\mathrm{BKZ}$ equations), as well as other integral constitutive equations in which the integrand can be expressed explicitly in terms of the strain. [The MeadLarson-Doi (1998) equation is not of this form and cannot be used within the formalism developed here.] The calculation is iterative, since the recoverable strain can only be determined after calculation of the total strain.

We demonstrate the approach using a separable single integral model

$$
\sigma=\int_{-\infty}^{t} M\left(t-t^{\prime}\right) h\left(I_{1}, I_{2}\right) C^{-1}\left(t, t^{\prime}\right) d t^{\prime} .
$$

$M(t)$ is the memory function

$$
M(t)=\sum_{i} \frac{G_{i}}{\lambda_{i}} \exp \left(-\frac{t}{\lambda_{i}}\right),
$$

and $C^{-1}$ is the Finger strain tensor. $h\left(I_{1}, I_{2}\right)$ is the damping function, which is a function of the first $\left(I_{1}\right)$ and second $\left(I_{2}\right)$ invariants of the strain tensor.

The deformation history is shown in Fig. 1. The flow with constant elongation rate $\dot{\varepsilon}$ starts at $t=0$. The filament undergoes rupture at $t=t_{0}$ and the stress falls immediately to zero, at which time recovery begins. The tensile stress $\left(\sigma_{11}-\sigma_{22}\right)$ for all $t>t_{0}$ is thus

$$
\begin{aligned}
\sigma_{11}-\sigma_{22}= & 0=\left(e^{-\varepsilon(t, 0)}-e^{2 \varepsilon(t, 0)}\right) \int_{-\infty}^{0} M\left(t-t^{\prime}\right) h\left(I_{1}, I_{2}\right) d t^{\prime} \\
& +\int_{0}^{t_{0}} M\left(t-t^{\prime}\right) h\left(I_{1}, I_{2}\right)\left[e^{-\varepsilon\left(t, t^{\prime}\right)}-e^{2 \varepsilon\left(t, t^{\prime}\right)}\right] d t^{\prime} \\
& +\int_{t_{0}}^{t} M\left(t-t^{\prime}\right) h\left(I_{1}, I_{2}\right)\left[e^{-\varepsilon\left(t, t^{\prime}\right)}-e^{2 \varepsilon\left(t, t^{\prime}\right)}\right] d t^{\prime}
\end{aligned}
$$

where 


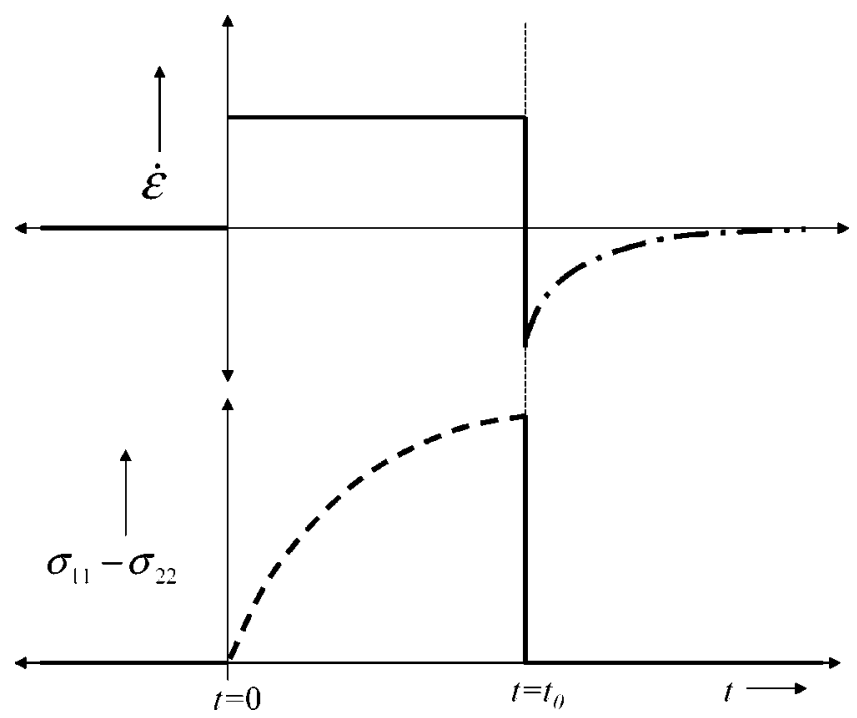

FIG. 1. Strain-rate and stress history of the filament. The system is deformed at a constant elongation rate until $t=t_{0}$, when the filament is cut or undergoes rupture.

$$
\varepsilon\left(t, t^{\prime}\right)=\int_{t^{\prime}}^{t} \dot{\varepsilon}\left(t^{\prime \prime}\right) d t^{\prime \prime}
$$

Equation (4) can be rearranged to the more convenient form

$e^{-\varepsilon(t, 0)}-e^{2 \varepsilon(t, 0)}$

$$
=-\frac{\int_{0}^{t_{0}} M\left(t-t^{\prime}\right) h\left(I_{1}, I_{2}\right)\left[e^{-\varepsilon\left(t, t^{\prime}\right)}-e^{2 \varepsilon\left(t, t^{\prime}\right)}\right] d t^{\prime}+\int_{t_{0}}^{t} M\left(t-t^{\prime}\right) h\left(I_{1}, I_{2}\right)\left[e^{-\varepsilon\left(t, t^{\prime}\right)}-e^{2 \varepsilon\left(t, t^{\prime}\right)}\right] d t^{\prime}}{\int_{-\infty}^{0} M\left(t-t^{\prime}\right) h\left(I_{1}, I_{2}\right) d t^{\prime}} .
$$

For the application here, we take a form for the damping function suggested by Wagner (1978) for elongational flows in which the Hencky strain is less than 2:

$$
h\left(I_{1}, I_{2}\right)=\exp \left[-m\left|\varepsilon\left(t, t^{\prime}\right)\right|\right]
$$

(The use of the absolute value in the argument is one of several proposals by Wagner to deal with flow reversal.) The special case $m=0$ is the multimode upper-convected Maxwell (or Lodge-Yamamoto) model.

The instantaneous recovery at $t=t_{0}$ is obtained analytically from Eqs. (3), (6), and (7) as

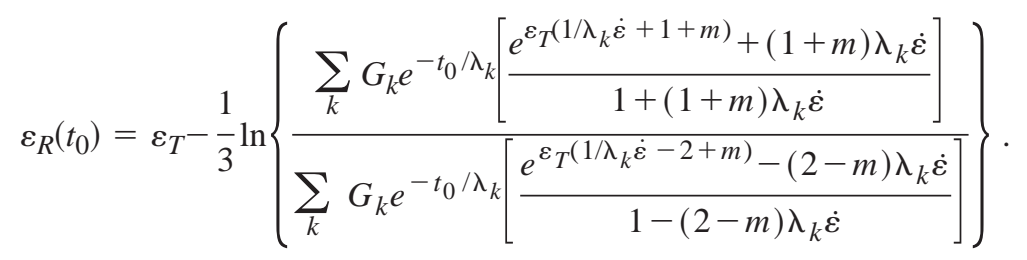

There is no delayed recovery for single-mode models. The ultimate recoil for multimode models is obtained by writing Eq. (6) for time $t+\Delta t$, numerically integrating the strain- 


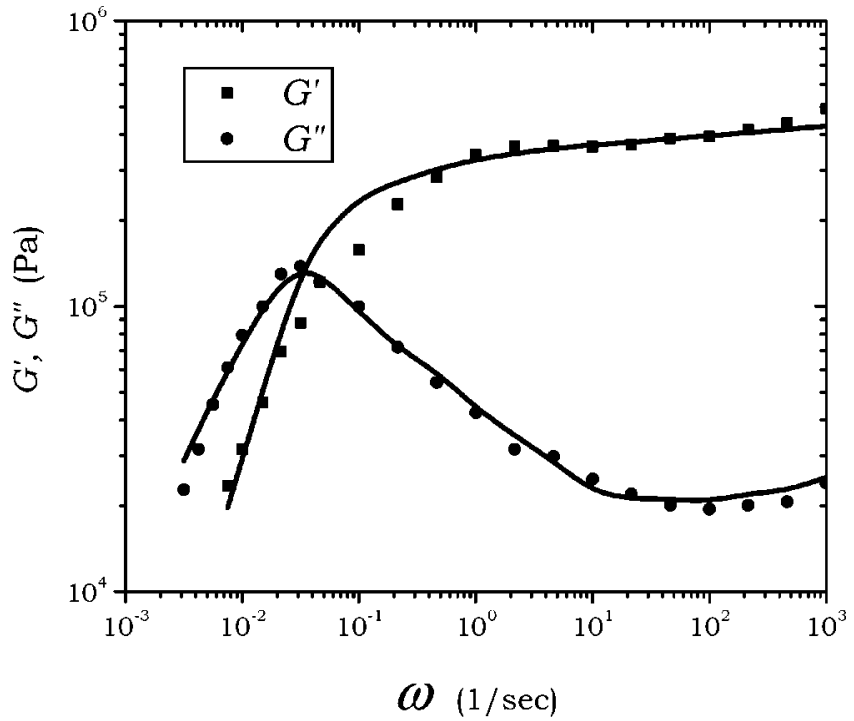

FIG. 2. Linear viscoelastic data for polyisoprene with a Molecular weight of 575,000 and a polydispersity of 1.02 at $25^{\circ} \mathrm{C}$ (Vinogradov et al., 1972). The lines are the fit with the memory function of Eq. (2) using the parameters in Table 1.

dependent integrals until time $t$ and using a simple Euler form for the integral between $t$ and $t+\Delta t$ to obtain the increment in strain. $\varepsilon\left(t, t^{\prime}\right)$ is a decreasing function of $t$ for $t$ $>t_{0}$.

\section{FIT TO RHEOLOGICAL DATA}

The data available for comparison with the rupture theory are for a polyisoprene with a molecular weight of 575000 and a polydispersity of 1.02 at $25^{\circ} \mathrm{C}$ [Vinogradov et al. (1972), (1975); Vinogradov (1975)]. The relaxation spectrum for this polymer was obtained by fitting the memory function Eq. (3) to small-angle oscillatory shear data [Vinogradov et al. (1972)]. Figure 2 shows the fit to $G^{\prime}$ and $G^{\prime \prime}$ using an Excel solver to minimize the sum of squares of data points, and the parameters are shown in Table I. The solver optimized the fit with seven relaxation modes. The low frequency data have not reached the terminal regime, and the longest relaxation time corresponds to a frequency that is below the range of the data. The data have a somewhat unusual shape in the neighborhood of the crossover frequency, and it is not possible to obtain good fits to both

TABLE I. Relaxation spectrum used for the calculations.

\begin{tabular}{ll}
\hline \hline$\lambda_{i}(\mathrm{~s})$ & $G_{i}(\mathrm{~Pa})$ \\
\hline 191 & 12600 \\
28.8 & 251000 \\
2.5 & 70000 \\
0.315 & 36000 \\
0.03 & 25000 \\
0.00443 & 25000 \\
0.00054 & 45000 \\
\hline \hline
\end{tabular}




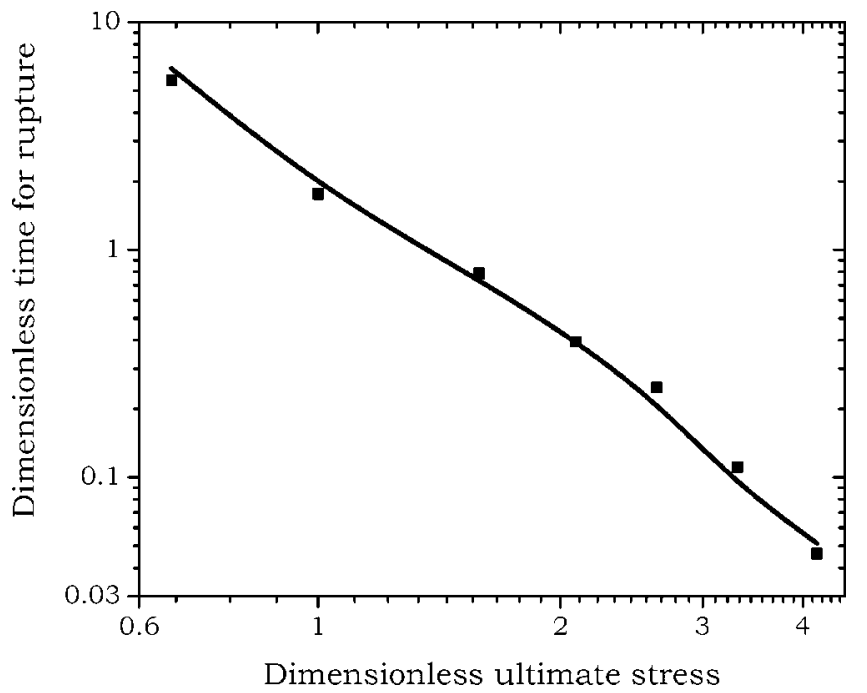

FIG. 3. Time to rupture as a function of ultimate (rupture) stress. Time is non-dimensionalized with reptation time and stress with plateau modulus. The points are the experimental data for polyisoprene at $25^{\circ} \mathrm{C}$ (Vinogradov et al., 1975). The fit of the Wagner model with $m=1.0$ to the data is shown by the solid line.

$G^{\prime}$ and $G^{\prime \prime}$ in this region. Similar results were obtained by Wissbrun (2003) using the Padé-Laplace method [Simhambhatla and Leonov (1993)].

The parameter $m$ in the Wagner model must be determined in a nonlinear experiment. This was done here by fitting the time to achieve the experimental breakup stress in one polyisoprene experiment, giving a value $m=1.0$, and then keeping the value of $m$ fixed for all other experiments. The calculated and experimental rupture times are plotted versus the experimental breakup stress in Fig. 3, and the fit is good. The calculated and experimental total strain and recoverable strain are plotted versus Weissenberg number in Fig. 4, where the reptation time was estimated as [Doi and Edwards (1986)] $\tau_{d}$ $=\left(12 / \pi^{2}\right)\left(\eta_{0} / G_{N}^{0}\right)=32 \mathrm{s.}{ }^{1}$ The total strain in Fig. 4 is derived from the rupture time in Fig. 3; the apparent difference in the quality of fit is a consequence of multiplication by the elongation rate and the use of a linear scale. The fit to the experimental data is adequate, but the spread between the experimental total and recoverable strains at the higher values of $W e_{\text {Rep }}$ seems unusually large and must be taken into account in considering the quality of the fit of the constitutive equation to the data. An accurate determination of ultimate recovery would have been extremely difficult to obtain in view of the presence of a relaxation mode of order $190 \mathrm{~s}$, and surface tension effects could have become important [Malkin, (2003)].

We also attempted to fit the experimental data in Figs. 3 and 4 with the Double Convective Reptation with Chain Stretch integral model of Ianniruberto and Marrucci (2001). The model predictions of stress are consistently lower than the experimental values for any given strain, and the model cannot predict the breakup conditions. In addition, the model predicts much less recovery for given total strains than observed experimentally or predicted by Eq. (1). Hence, it is not an appropriate rheological model for these calculations.

${ }^{1}$ The value of $G_{N}^{0}$ used in Joshi and Denn (2003) was $0.55 \mathrm{MPa}$, based on data in Fetters et al. (1994). Here, for consistency, we use the value $0.38 \mathrm{MPa}$ from the data in Fig. 2. This change results in different values of $N, N_{e}$, and $\tau_{d}$, and a small displacement of the line representing Eq. (1) in the figures. 


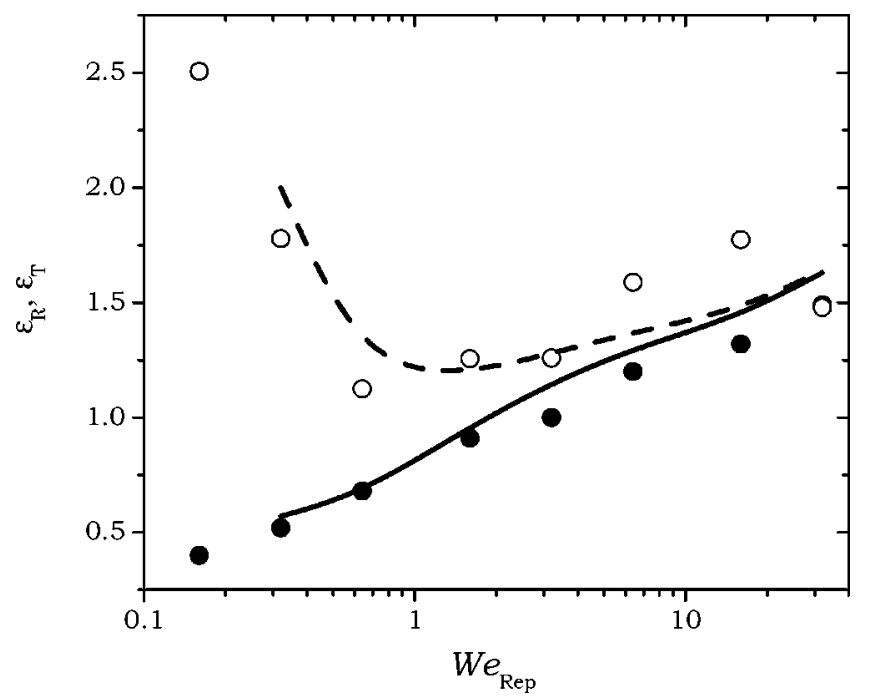

FIG. 4. Total strain (open circles) and recoverable strain (filled circles) as functions of Weissenberg number for polyisoprene at $25^{\circ} \mathrm{C}$ [Vinogradov et al. (1975)]. The dashed line is the total strain obtained from the time to rupture in Fig. 3. The solid line is the corresponding prediction of recoverable strain from the Wagner model.

\section{PREDICTION OF RUPTURE}

We now show the a priori calculation of breakup using Eq. (1) and the Wagner model with $m=1.0$. Unlike the preceding section, where we used the experimental failure stress as the input, we now use the scaling theory to predict the critical recoverable strain for a given elongation rate, from which we compute the total strain at failure. The light solid line in Fig. 5 is Eq. (1) for the recoverable strain, calculated from the scaling theory with $\beta=1$, and the light broken line is the corresponding total strain. The scaling theory with $\beta=1$ captures the trend of the experimental recoverable strain, but it consistently underpredicts the data by about 0.25 strain units. The corresponding prediction of the total strain at failure thus fails to capture the trend of the data at $W e_{\text {Rep }}$ less than unity.

The heavy solid line in Fig. 5 is Eq. (3) with $\beta=2.5$; this value of $\beta$ was chosen so that the scaling theory approximates the recoverable strain data at failure. The corresponding heavy broken line is the prediction of the total strain at rupture. The calculated total strain exhibits the important qualitative features of Vinogradov's second and third regimes, although the calculation generally underpredicts the data.

\section{CONCLUSION}

Rupture can be predicted in both the viscoelastic and rubbery regimes of elongational flow of entangled melts using the scaling theory, Eq. (1), to estimate the critical recoverable strain. The Wagner model captures the important qualitative features of the experimental data of Vinogradov and co-workers, and the quantitative agreement is as good as can reasonably be expected with so simple a model. The experimental data themselves show a large degree of variability, especially at higher Weissenberg numbers, and a larger data set is needed for an adequate test of the approach. 


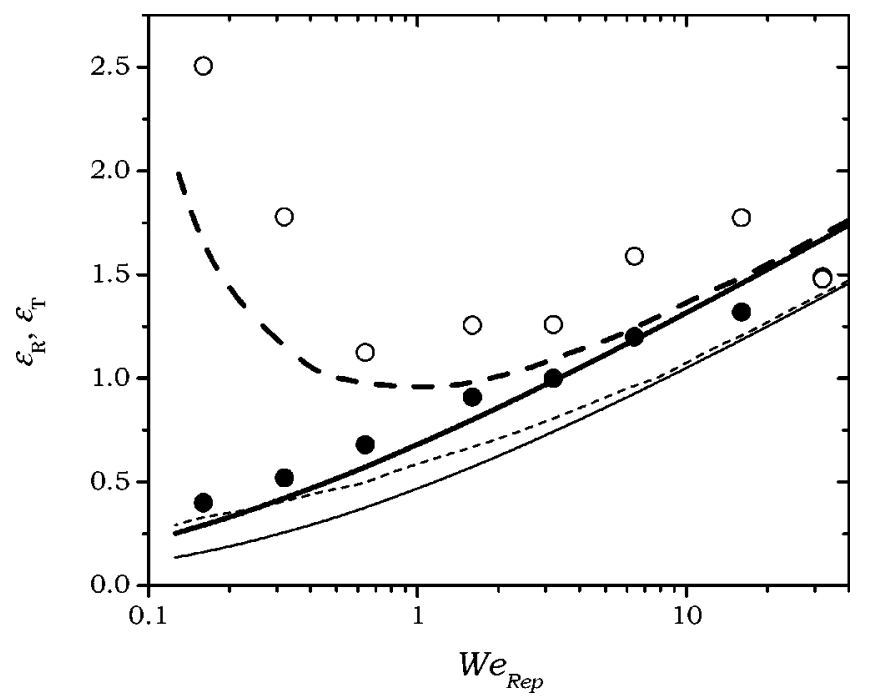

FIG. 5. Total strain and recoverable strain at $25^{\circ} \mathrm{C}$ as functions of Weissenberg number. Symbols are as in Fig. 4. The light solid line is the prediction of the scaling model, Eq. (1), with $\beta=1$, and the light broken line is the corresponding total strain predicted from the Wagner model. The heavy continuous line is the prediction of Eq. (1), with $\beta=2.5$, and the heavy broken line is the corresponding total strain predicted from the Wagner model.

\section{ACKNOWLEDGMENT}

We are grateful to Hans-Martin Laun, João Maia, Alex Malkin, and Kurt Wissbrun for stimulating discussions that greatly enhanced our understanding of the issues involved in this work.

\section{References}

Doi, M., and S. F. Edwards, The Theory of Polymer Dynamics (Clarendon, Oxford, 1986), p. 229.

Fetters, L. D., D. J. Lohse, D. Richter, T. A. Witten, and A. Zirkel, "Connection between polymer molecular weight, density, chain dimensions, and melt viscoelastic properties," Macromolecules 27, 4639-4647 (1994).

Ghijsels, A., C. H. C. Massardier, and R. M. Bradley, "Brittle melt rupture phenomena in polymer processing-An overview," Int. Polym. Process. 12, 147-154 (1997).

Ianniruberto, G., and G. Marrucci, "A simple constitutive equation for entangled polymers with chain stretch," J. Rheol. 45, 1305-1318 (2001).

Joshi, Y. M., and M. M. Denn, "Rupture of entangled polymeric liquids in elongational flow," J. Rheol. 47, 291-298 (2003).

Joshi Y. M., and M. M. Denn, "Failure and recovery of entangled polymer melts in elongational flow," in Rheology Reviews 2004, edited by K. Walters and D. Binding (British Society of Rheology, Aberystwyth, 2004).

Malkin, A. Ya. [personal communication (2003)].

Malkin, A. Ya., and C. J. S. Petrie, "Some conditions for rupture of polymer liquids in extension," J. Rheol. 41, 1-25 (1997).

Mead, D. W., R. G. Larson, and M. Doi, "A molecular theory for fast flows of entangled polymers," Macromolecules 31, 7895-7914 (1978).

Simhambhatla, M., and A. I. Leonov, "The extended Padé-Laplace method for efficient discretization of linear viscoelastic spectra," Rheol. Acta 32, 589-600 (1993). 
Vinogradov, G. V., "Viscoelasticity and fracture phenomenon in uniaxial extension of high-molecular linear polymers," Rheol. Acta 14, 942-954 (1975).

Vinogradov, G. V., A. Ya. Malkin, V. V. Volosevitch, V. P. Shatalov, and V. P. Yudin, "Flow, high-elastic (recoverable) deformations and rupture of uncured high molecular weight linear polymers in uniaxial extension,” J. Polym. Sci., Polym. Phys. 13, 1721-1735 (1975).

Vinogradov, G. V., A. Ya. Malkin, Yu. G. Yanovskii, E. K. Borisenkiva, B. V. Yarlykov, and G. V. Berezhnaya, "Viscoelastic properties and flow of narrow distribution polybutadienes and polyisoprenes," J. Polym. Sci., Part A-2 10, 1061-1084 (1972).

Wagner, M. H., "A constitutive analysis of uniaxial elongational flow data of a low-density polyethylene melt," J. Non-Newtonian Fluid Mech. 4, 39-55 (1978).

Wissbrun, K. F. [personal communication (2003)]. 